\title{
Testing cognitive models of visual attention with fMRI and MEG
}

\author{
Paul Downing a,*, Jia Liu ${ }^{\mathrm{b}}$, Nancy Kanwisher ${ }^{\mathrm{b}, \mathrm{c}}$ \\ a School of Psychology, Centre for Cognitive Neuroscience, University of Wales, Bangor, Gwynedd LL57 2AS, UK \\ ${ }^{\mathrm{b}}$ Department of Brain and Cognitive Sciences, MIT, Cambridge, MA 02139, USA \\ ${ }^{\mathrm{c}}$ MGH-NMR Center, 149 13th Street, Charlestown, MA 02129, USA
}

\begin{abstract}
Neuroimaging techniques can be used not only to identify the neural substrates of attention, but also to test cognitive theories of attention. Here we consider four classic questions in the psychology of visual attention: (i) Are some 'special' classes of stimuli (e.g. faces) immune to attentional modulation?; (ii) What are the information units on which attention operates?; (iii) How early in stimulus processing are attentional effects observed?; and (iv) Are common mechanisms involved in different modes of attentional selection (e.g. spatial and non-spatial selection)? We describe studies from our laboratory that illustrate the ways in which fMRI and MEG can provide key evidence in answering these questions. A central methodological theme in many of our fMRI studies is the use of analyses in which the activity in certain functionally-defined regions of interest (ROIs) is used to test specific cognitive hypotheses. An analogous sensor-of-interest (SOI) approach is applied to MEG. Our results include: evidence for the modulation of face representations by attention; confirmation of the independent contributions of object-based and location-based selection; evidence for modulation of face representations by non-spatial selection within the first $170 \mathrm{~ms}$ of processing; and implication of the intraparietal sulcus in functions general to spatial and non-spatial visual selection. (C) 2001 Elsevier Science Ltd. All rights reserved.
\end{abstract}

Keywords: Cognitive models; fMRI; MEG

\section{Introduction}

Without attention, the contents of our awareness would be strictly determined by the stimuli impinging on our senses. With attention, we can select which stimuli will be analyzed in detail and will be allowed to guide our behavior, making us active participants in the construction of our own perceptual experience. Efforts to understand attention have recruited every method in the toolbox of cognitive neuroscience. This enterprise has led to a wealth of new insights into questions that have been at the heart of attention research since the 1950s and 1960s. In this chapter we review recent work from our laboratory using fMRI and MEG to address four key issues concerning visual selective attention.

\footnotetext{
* Corresponding author. Tel.: + 44-1248-382-159; fax: + 44-1248382-599.

E-mail address: p.downing@bangor.ac.uk (P. Downing).
}

\subsection{Four questions about visual attention}

The earliest research in selective attention asked how processing of a stimulus was affected by directing attention toward that stimulus versus away from it. For example, when subjects listen to a message played into one ear while an ignored message is played simultaneously into the other ear, what kinds of representations are extracted from the ignored message [11]? We discuss here fMRI and MEG studies that address an analogous question for the case of vision. These techniques allow direct measurement of the neural processing of a specific visual stimulus as a function of whether that stimulus is attended or not. We focus in particular on how attention modulates the processing of faces. Faces are at the heart of our investigations for two reasons. First, recent work in fMRI, ERP, and MEG (discussed below) provides sensitive and selective measures of face processing in humans, making them ideal from a methodological standpoint. And second, owing to their high familiarity, relevance, and likely evolutionary import, faces may represent a special class of stim- 
uli for humans, perhaps with priority over the guidance of attention [32,55,84,85].

Our second question concerns the information units that attention operates on. Most early theories $[31,49,68,69,79]$ emphasized spatial location as the medium of attention. According to these models, attention selects locations, resulting in the enhancement of all the information occupying the attended location relative to information occupying unattended locations. However, more recent evidence has demonstrated that the organization of spatial information into perceptual groups, or objects, also strongly influences the allocation of attention $[24,42]$. While some of the earlier work on location- and object-based attention regarded them as mutually-exclusive alternatives, most current theories of attention (e.g. [21,80]) can accomodate the predictions of both. We review two fMRI studies that separately examine location- and object-based selection, confirming the key predictions of each. These results lend additional support to the idea that both mechanisms of selection must co-exist, and suggest approaches for further research into how they interact.

Third, attentional modulation of a stimulus could in principle take place at any stage in visual processing; how early does it actually occur when the stimuli are faces? While this question has been addressed in the domain of spatial selection using ERP [27], we briefly describe an MEG experiment demonstrating relatively early (e.g. at a latency of $170 \mathrm{~ms}$ ) modulation of face processing that can not be due to spatial orienting.

Finally, a distinction can be made between attentional mechanisms that operate on spatially distributed stimuli, and nonspatial mechanisms that operate on temporally-distributed stimuli $[18,56,76]$. The final study we review [88] asks whether the control of spatial and non-spatial attention invoke common cortical structures, and by implication common cognitive mechanisms.

\subsection{Application of neuroimaging techniques to theories of attention}

Many researchers have emphasized the capabilities of fMRI and ERP/MEG to measure the neural correlates of cognitive function with high spatial and temporal precision, respectively. However, there is less agreement on the question of whether these neuroimaging techniques can provide decisive tests of psychological theories. We argue here that they can, and we use recent studies from our lab to illustrate our approach to this problem (see also $[10,14,16,28,36,47,48,54,73-75]$ ).

Our studies generally proceed in two steps. First, we capitalize on the spatial and temporal resolution of fMRI and MEG to isolate and characterize neural markers of stimulus- or task-specific processes. In the second step, these neural markers are then used as dependent measures, to test cognitive hypotheses about selective attention. More specifically, several of the fMRI studies reviewed here make use of two cortical regions of interest (ROIs) identified from previous fMRI research: the fusiform face area (FFA), which responds selectively to faces [43], and the parahippocampal place area (PPA), which responds selectively to places and houses [29,30]. Activity in each of these regions is used as a neural marker of processing of its preferred stimuli. The precise characterization of the computations carried out in each of these regions is not yet clear, and remains an active area of ongoing research and debate $[44,46,78]$. However, the selectivity of each region for its 'preferred stimulus' (faces for the FFA, and places or houses for the PPA) has been replicated in many laboratories $[1,39,58]$ and is not in dispute. For the present purposes of using the activity in each of these regions as a marker for the processing of a specific stimulus, the selectivity of the response in each region is sufficient. It is unlikely that the ultimate understanding of the functions of these regions will alter the conclusions about visual attention that we draw from the studies described here.

Previous studies have identified a component of the ERP response that occurs at a latency of $170 \mathrm{~ms}$ over occipitotemporal areas (the 'N170'), showing a substantially greater response amplitude to visually presented faces than to objects from other categories $[2,3,7,40,59,72]$. An apparently analogous response component has also been observed with MEG [34,51] that is similarly selective for faces and that occurs at the same latency (the 'M170'). Like the response of the FFA as measured with fMRI, the M170 response generalizes to a variety of face stimuli including profile faces, animal faces, and line drawings of faces, but not to control stimuli such as houses and other objects [51] (see Fig. 1). Indeed, Halgren et al. [34] have suggested that the two measures derive from the same neural source. Whether or not this turns out to be true, the selectivity and timecourse of the M170 complement the FFA as a measure of face-selective processing. While fMRI responses presumably reflect the sum of processing at all latencies, the M170 reflects processing that occurs at or before this relatively short latency.

We discuss here an MEG 'sensor-of-interest' (SOI) approach that is analogous to the fMRI region of interest approach described above. We conduct an initial 'localizer' test in each subject to find the individual sensors in each hemisphere that show the strongest face selectivity over occipitotemporal sites (typically T5/T6 on the 10-20 ERP system). The amplitude and latency of the M170 at these sensors of interest are then used as the dependent measures to test for attentional modulation of face processing.

In the studies described below, ROIs (or SOIs in the case of MEG) are defined individually in each subject, 
in an independent series of scans as described in previous reports $[29,51,66]$. Subjects are then tested on further scans in which the preferred and non-preferred stimuli for these ROIs/SOIs are presented while attention is manipulated with task instructions. An increased response in a given $\mathrm{ROI} / \mathrm{SOI}$ is taken as an indicator of increased processing of the preferred stimulus (for that ROI/SOI). We show below how this careful characterization of the response properties of a cortical region (or an MEG response), combined with behavioral manipulations of attention, can provide powerful tests of psychological hypotheses.
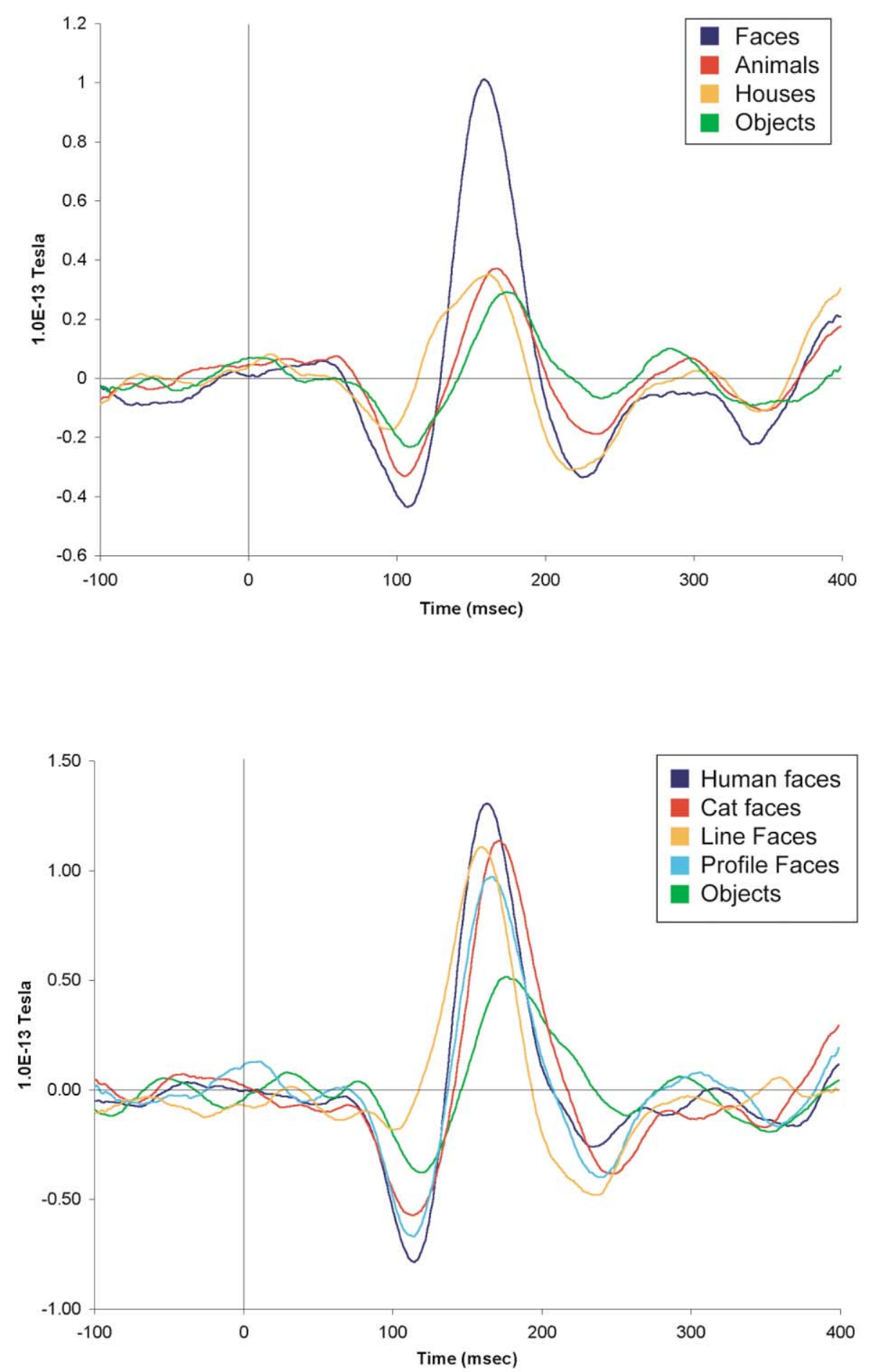

Fig. 1. The M170 response, averaged across subjects, to a variety of stimulus types. Top: The M170 appears specifically to reflect face processing, rather than a more general process such as subordinate-level categorization, or processing of anything animate. Bottom: The M170 response generalizes across faces that vary in species, image format, and viewpoint [52]. 


\section{Examples of recent neuroimaging studies of attention}

Next we present four studies from our lab that address the questions outlined above. Our purpose is not to provide a comprehensive review of the growing literature in this area, but simply to illustrate some of the ways in which we have used neuroimaging to answer cognitive questions about attention.

\subsection{Fate of unattended stimuli}

In most real-world visual scenes, multiple objects are in view at any one time. When a subset of these objects is selectively attended, our awareness of the remaining stimuli is reduced. But what exactly has happened to the representations of the unattended items? Neuroimaging techniques are particularly useful in measuring the cortical response to ignored stimuli. Behavioral approaches to this question typically rely on some kind of overt response to unattended stimuli [e.g. [83]], necessarily drawing some attention to them [see [26] for discussion]. In contrast, neuroimaging studies can measure the neural response to truly irrelevant stimuli without affecting the subjects' deployment of attention, a fact ERP studies have capitalized on for decades $[35,36,54]$.

Wojciulik et al. [87] asked whether processing of faces can be modulated by selective attention. It has been argued that faces are processed by a specialized neural system separate from that involved in other forms of object recognition. On some accounts this specialized module might be expected to be engaged by faces obligatorily, regardless of their current relevance (e.g. [32]). Wojciulik et al. [87] tested this hypothesis by comparing the responses of the FFA to faces when they were task-relevant to when they were task-irrelevant, while holding the retinal stimulus constant. Subjects were shown a series of displays containing two faces and two houses arranged in the manner shown in Fig. 2. On some presentations the two houses were identical, and on others the two faces were identical. In separate blocks, subjects attended to the faces or houses and made a same-different judgment on them, ignoring the irrelevant stimuli. If faces are processed to the same extent regardless of how the subject allocates attention, then FFA activity evoked by the compound stimulus should be equal in the attend-face and attend-house conditions. In contrast to this prediction, Wojciulik et al. [87] found instead that FFA activity was significantly higher in attend-face blocks compared to attendhouse blocks.

This study serves two purposes for the present discussion. First, like other studies using single-unit neurophysiology [53,60,61], ERPs [27,36,54], and brain imaging $[5,14,65,73]$, it demonstrates the effects of attention on the internal representation (or processing) of

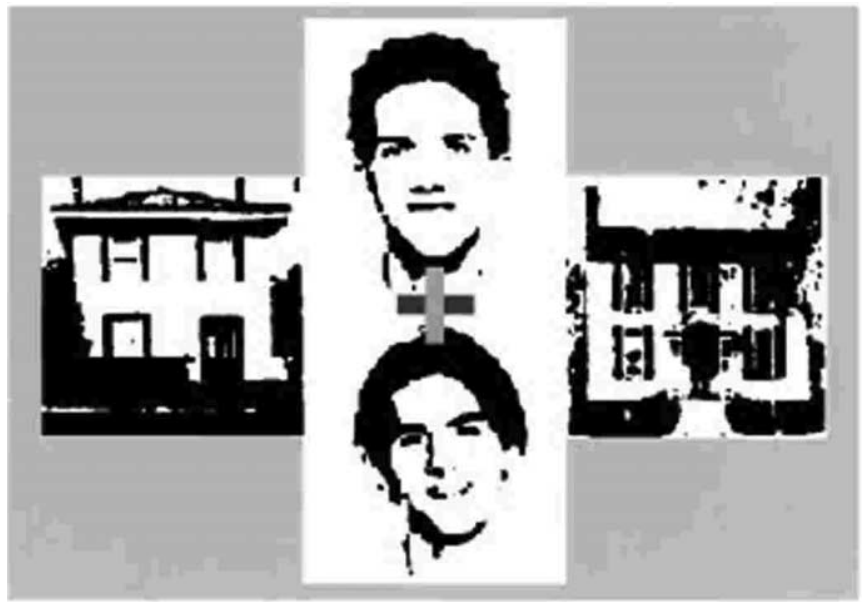

Fig. 2. Illustration of stimuli from Ref. [87]. In separate blocks, subjects performed a matching task on either faces or houses.

a stimulus. When faces were task-irrelevant, their processing, as revealed by activity in the face-specific FFA, was reduced compared to when they were attended. Second, activation in the FFA was not uniformly high whenever a face was present, but rather depended on attention. Although evidence has accumulated for the unique status of faces in visual recognition, faces are evidently not so special as to be immune to the effects of attention (c.f. [84]).

The attentional modulation observed in Wojciulik et al. [87] can be explained in at least two ways, corresponding to two kinds of attentional selection. First, subjects might have used 'feature-based' selection to activate representations of the visual features present in faces (or houses) (c.f. [82]). A second possibility is that attention was allocated instead to the spatial locations in which the perceptually-relevant information was presented. In Wojciulik et al.'s [87] experiment, the faces were in the same position for an entire block, and switched positions with the houses only between blocks. As a result, by attending to either a horizontal or vertical region of space surrounding fixation, subjects could restrict attention to encompass face information or house information exclusively. Thus while the Wojciulik et al. [87] study demonstrates modulation of representations by attention, it does not determine the units of information on which attention operates. The following studies were intended to directly address this issue.

\subsection{Units of selective attention}

The psychological literature on attention has distinguished between location-based, feature-based, and object-based forms of selection. While at times they are cast as mutually-exclusive alternatives, current theories of attention can accomodate all three. For example, 
Desimone and Duncan's biased-competition model [21], which emphasizes whole objects as the units of selection, suggests that increased activation of a visual feature will tend to provide a competitive bias to objects sharing that feature over those that do not, a form of feature-based selection. Similarly, Treisman's updated version of feature integration theory [80], while it emphasizes the special role of location, includes featurespecific inhibition across the visual array, as well as selection of inputs specified by the contents of an object file [41].

Can these three components of attention be studied independently? Each kind of selection will have different consequences for the kinds of irrelevant information that will tend to be selected as a result of attending to relevant information. Here we focus on the contrast between location- and object-based selection. Locationbased attention will tend to select both relevant and irrelevant stimuli at the attended location. On a strict interpretation of this idea, the grouping of stimuli into objects should not influence the allocation of attention. In contrast, however, more recent evidence (e.g. $[4,23,24,42])$ has illustrated the importance of perceptual groups, and particularly whole objects, in guiding selection. A critical implication of this idea is that attention to one part or attribute of an object will entail selection of the whole object, relative to other objects in the scene $[20,25,86]$.

We have conducted fMRI experiments to test these predictions of both object- and location-based selection. Our goal was to create stimuli and tasks that distinguish these mechanisms by their differing consequences for the processing of task-irrelevant information. As in Wojciulik et al. [87], each experiment used stimulus-selective regions of visual cortex as a measure of attentional modulation.

\subsubsection{Object-based attention}

O'Craven et al. [66] tested for the following predicted consequence of object-based attention: attending to one attribute of an object should enhance processing of the other attributes of that object, compared to attributes of irrelevant objects (even those appearing at the attended location). To distinguish the effects of objectbased selection from location- and feature-based selection, stimuli were created with two objects transparently overlapping at the same location (see Fig. 3). One of the two objects in each display had a second visual attribute, namely low-amplitude oscillating motion.

Location-based attention would not efficiently select just one of the two objects in this figure; attention to the face, for example, would entail selection of the house as well, because both appear in the same location. Feature-based selection would allow enhancement of one of the three dimensions (e.g. face, house, or motion) present in the stimulus, relative to the other two. For object-based selection alone, however, attention directed to one of the stimulus dimensions (e.g. the motion) should lead to selection of that entire object, including its irrelevant dimension (e.g. the face) but not the other object at the same location (e.g. the house).

These predictions were tested by measuring activity in the FFA (which responds more strongly to faces than houses), and the PPA (which responds more strongly to houses than faces). Importantly, the houses and faces themselves were never task-relevant. In different scans, subjects' attention was directed either to the direction of motion of the moving stimulus, or to the position of the static item, which was displaced slightly off of fixation in one of four directions. In a mixed, event-related design, half of the time the attended item was a face and the ignored item a house, and half of the time this arrangement was reversed. As a result, subjects could not use the task-irrelevant dimension (the face or house), to predict anything about the task-relevant dimension, and thus had no motivation to attend to it.

The results of this study (see Fig. 4) showed that when subjects attended to motion, the response was higher in the PPA when it was the house that moved compared to when the face moved; the opposite pattern was found in the FFA. In a significant triple interaction, each of these findings for both the FFA and PPA reversed when subjects directed their attention instead

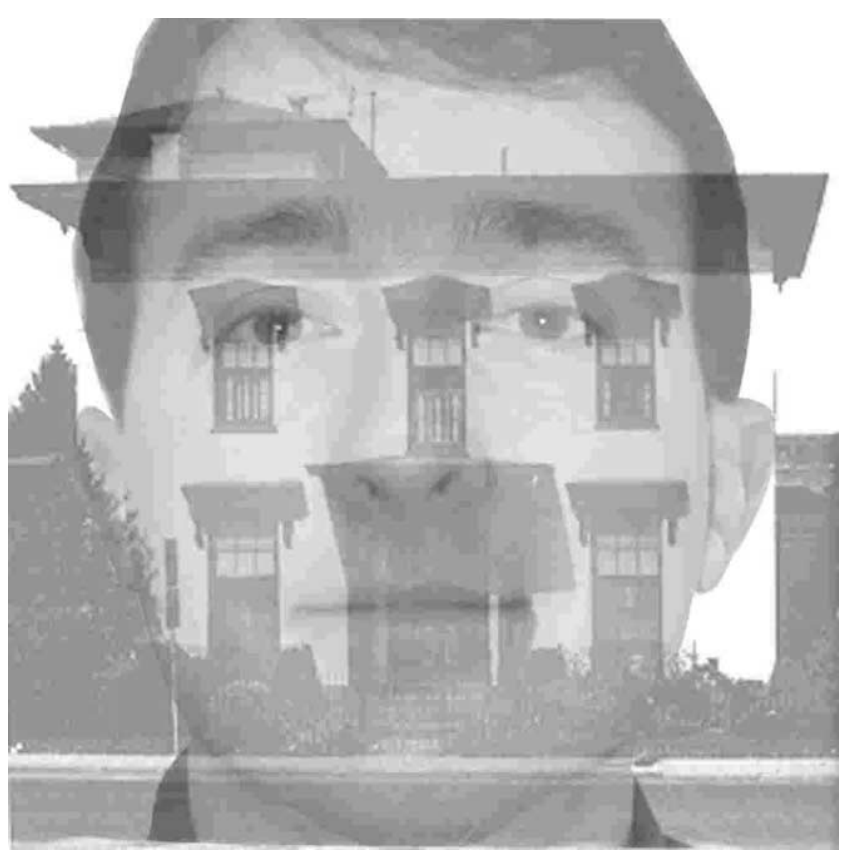

Fig. 3. Illustration of stimuli from Ref. [66]. On each trial, either the house or the face oscillated slightly along one axis, transparently, while the other remained stationary. Subjects attended to either the direction of motion or to the position (relative to fixation) of the stationary item. 
FFA

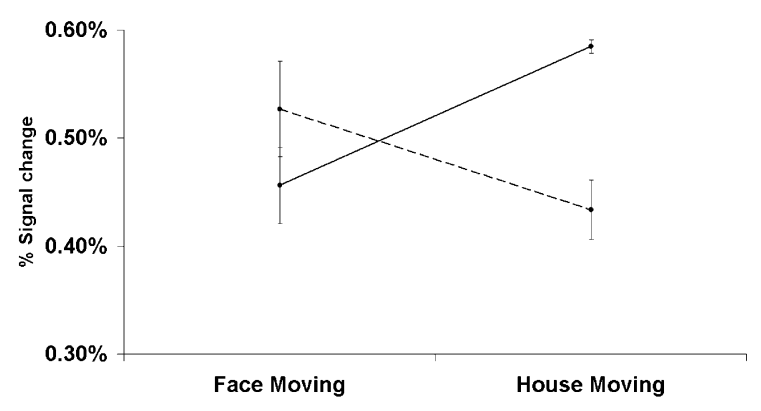

PPA
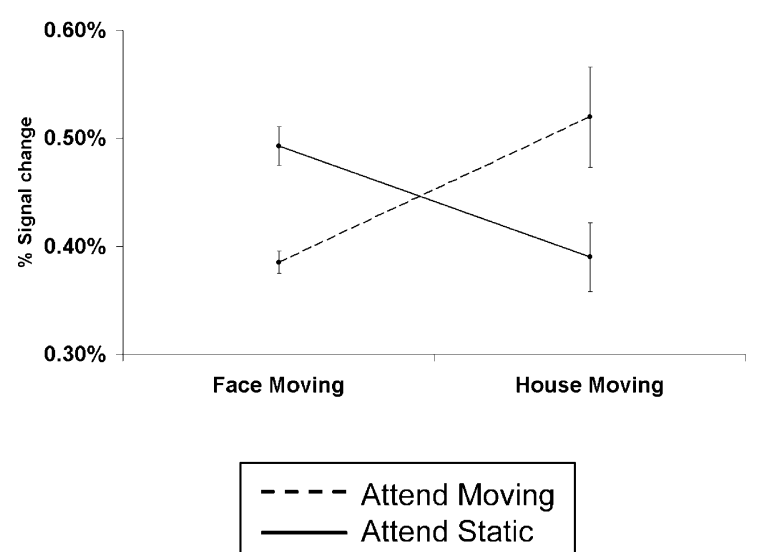

Fig. 4. Means of the peaks of grand-averaged event-related fMRI responses in the FFA and PPA, from Ref. [66]. Error bars reflect the standard error of the mean after correcting for between-subject variance. In both areas, the response was greater when attention was directed to another feature (location or motion) of the preferred stimulus for that area, compared to the non-preferred stimulus. The three-way interaction of brain area (FFA or PPA), moving stimulus (face or house), and attended dimension (motion or location) was significant, $P<0.05$.

to the position of the non-moving item. Thus the task-irrelevant features in each display received more attention when they were associated with the attended object, compared to an ignored object at the same location, implicating object-based selection uniquely.

How is it possible for the features of two objects transparently occupying the same location to be segregated? It may be that a high-level representation of the to-be-attended dimension feeds back to lower-level systems, highlighting those features consistent with the target object [50]. In the case of transparency, parts of the compound image will be relatively dominated by one object or the other, while other parts will be an inextricable blend of the two. Top-down guidance of attention by an existing object representation would allow selection and grouping of the unambiguous features. Because of the fine spatial scale on which features are interleaved in transparent figures, it seems likely that only early visual areas such as V1, where neurons have small receptive fields, would have sufficient resolution to accomplish this task. On this account, spatial location necessarily plays a role in selection, but the guidance of attention is accomplished by an object representation. It remains a puzzle for any model of attention to describe in detail the mechanisms that solve the binding problems $[8,9,81]$ revealed in displays like the one used here.

\subsubsection{Location-based attention}

O'Craven et al.'s [66] study was designed specifically to isolate the contributions of object-based selection. An analogous test for location-based effects would require measuring the response to an unattended stimulus as a function of whether it is at the location of an attended item, compared to when it is far from the attended item [12,13,37]. Here we report a new experiment [22] that met this criterion, and additionally ruled out possible contributions of object- and feature-based selection.

Attention was drawn to one of two locations with a task of reporting the orientation of one oval of a given color while ignoring an oval of a different color at another location (see Fig. 5). Each of the colored ovals (one to the left of fixation and one to the right) was superimposed on a task-irrelevant face or house. Location-based selection should lead to enhancement of the task-irrelevant face or house stimulus presented at the attended location, compared to when the same stimulus appears at the unattended location. To test this prediction we used the fMRI response in the FFA and PPA as a measure of the processing of the face and house stimuli.

Five subjects (three naive, two authors [NK and PD]) participated in the experiment. In a mixed, event-related design, subjects were presented with displays like those illustrated in Fig. 5. On each trial, one red and one green oval were presented flanking a fixation point. Each colored oval appeared equally often to the left and right of fixation. On each trial, an irrelevant stimulus, either a face or a house, was also presented at the location of each oval. These stimuli were either both faces (1/4 of trials), both houses (1/4 of trials), or one of each ( $1 / 2$ of trials). For trials in which a face and a house were both presented, each stimulus type appeared equally often at the same location as the red or green oval. On each trial, each oval was randomly determined to be either vertical or horizontal.

Within each session for each subject, separate scans were collected to identify the FFA and PPA as reported previously $[29,43]$. Each subject was then run on four scans of the attentional experiment. In two of the scans, subjects attended to and reported the orientation of the green ovals, and in the other two scans they reported on the red ovals. Each scan consisted of 162 trials. 
There were 18 fixation-only baseline trials, and 18 trials in each of eight conditions formed by crossing the arrangement of the colored ovals (green on left or right) with the types of irrelevant stimuli (two faces, two houses, a house on the left with a face on the right, or a face on the left with a house on the right). The ordering of trials was random, with the constraint that trials from each condition (including baseline) were immediately preceded equally often by trials from every condition. A different trial sequence was used for each scan. Scans began and ended with a 16-s fixation-only epoch.

Each trial lasted 2 s. On non-fixation trials, the display was presented for $167 \mathrm{~ms}$, in order to prevent subjects from refixating the display while it remained on the screen. Houses and faces were randomly selected on each trial from a set of eight items in each category. Subjects responded to the orientation of the ovals by pressing one of two buttons on a keypad.

Scanning was conducted on the 4T Varian scanner at the University of Western Ontario. A custom surface coil was used to increase signal to noise in posterior visual cortex. Seven slices ( $3 \mathrm{~mm}$ in-plane resolution; 6 or $7 \mathrm{~mm}$ thickness) were collected covering the occipitotemporal regions of the FFA and PPA. Data were acquired at TR $=1 \mathrm{~s}$ to efficiently measure the event-related response.

The fMRI signal from the FFA and PPA was extracted for each scan individually. Twelve time-points (12 s) of data were averaged by condition beginning from the onset of each trial. The data were converted to percent signal change relative to fixation trials, and mean percent signal changes were then calculated for each condition of interest for each subject.

The critical predictions concerned trials in which one face and one house were present. For these conditions, the stimulus was held constant - one preferred and one non-preferred stimulus for each of the two ROIs - and any modulation of response must be due to the alloca- tion of attention to the task-relevant ovals in each display.

For each ROI, we compared the average evoked response from trials in which the preferred object for that ROI occupied the attended location against trials in which the non-preferred object occupied that location. The grand mean evoked responses for the FFA and PPA are shown in Figs. 6 and 7, respectively. In each ROI, the evoked response was larger when its preferred stimulus appeared at the attended location compared to when it appeared at the unattended location. This observation was quantified by performing a $2 \times 2 \times 2$ ANOVA with ROI (FFA vs. PPA), stimulus at attended location (preferred vs. non-preferred) and time bin (the average response at seconds $0-3$ vs. seconds 4-7 after stimulus presentation) as factors. We reasoned that any increased activation to preferred over non-preferred stimuli should only be observed at the peak of the response (which is typically 4-7 s after stimulus onset), and not within the first few seconds, due to the sluggishness of the hemodynamic response.

There was a significant main effect of stimulus condition (preferred vs. non-preferred at attended location), $F(1,4)=8.8, P<0.05$. This effect was qualified by an interaction of this factor with time bin, $F(1,4)=8.4$, $P<0.05$. Over the mean of the first four time points, there was no difference between preferred $(M=0.03 \%$ signal change) and non-preferred stimuli $(M=0.04 \%)$, $F<1$, ns, showing that there were no differences in baseline response across conditions. In contrast, over the mean of the second four time points, there was a significantly higher response to the preferred stimulus $(M=0.25 \%)$ compared to the non-preferred stimulus $(M=0.18 \%), F(1,4)=9.2, P<0.05$. The three-way interaction did not reach significance, $F(1,4)=2.1$, ns, indicating that this effect did not differ significantly between the FFA and the PPA. No other theoreticallyrelevant effects were significant.

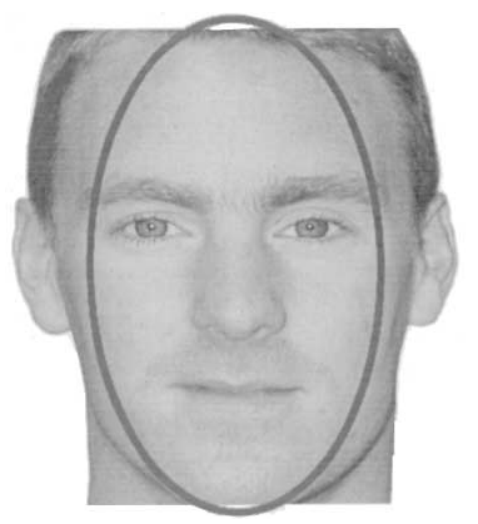

Fig. 5. Illustration of stimuli from an fMRI test of location-based attention [22] (ovals were red or green in the actual stimulus). Subjects attended to the orientation of either the red or green ovals, in separate blocks. In a mixed, event-related design, a face or house appeared unpredictably at the same location as the attended and ignored ovals. The faces and houses served as attentional probes, and were themselves never task relevant. 


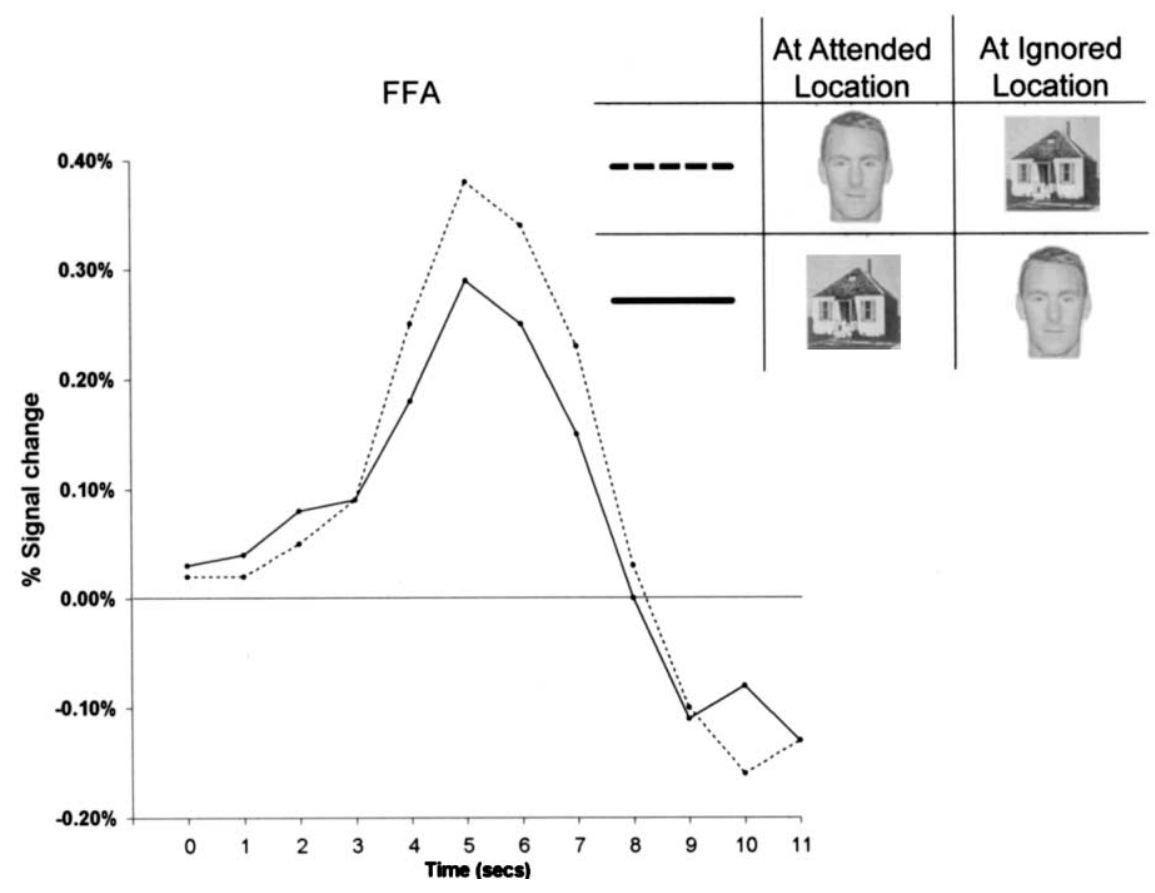

Fig. 6. Results from an fMRI test of location-based attention [22]. The mean event-related evoked response in the FFA was greater when a face occupied the same location as the attended oval, as compared to a house.

These results would be expected only from a location-based selection mechanism. On trials in which subjects attended a location that also contained a face, activity was greater in the FFA than on trials in which a house appeared at the attended location. Conversely, activity in the PPA was greater when a house appeared at the attended location compared to a face. Critically, the face and house stimuli were never relevant to the task, so these attentional effects are unlikely to reflect any intentional strategy adopted by the subject. Additionally, the markers of attentional modulation (the faces and houses) bore no particular featural overlap with the task-relevant items (the colored ovals); thus these results would not be predicted on the basis of feature-based selection. Likewise, the task-relevant and irrelevant information consisted of perceptually distinct objects; thus object-based selection would not produce the kind of modulation observed here.

Finally, it is worth emphasising that, owing to the design of both this experiment and that of O'Craven et al. [66], the attentional modulation observed in these studies is unlikely to be due to a baseline increase in activation of cortical areas selective for faces and houses (see [48]). Subjects could not predict from trial to trial whether a house or face would share the same object as the attended feature [66] or appear at the attended location (in the present study). Furthermore, attentional effects were observed in cortical areas measuring processing of task-irrelevant stimuli. Thus, while the tasks used may have led to baseline increases in task-related areas (e.g. area MT, in the motion task of
[66]), this could not explain the transient increases in activity seen in the cortical areas (FFA and PPA) that revealed the resulting spread of attention to irrelevant stimuli.

\subsection{The time course of selection}

Imaging techniques such as PMRI and PET that rely on blood flow to measure neural activity have poor temporal resolution and therefore tend to average together all of the activity of an entire processing episode. Thus, they will generally not be sensitive to the distinction between initial processing of a stimulus and later top-down aspects of stimulus processing [50,57]. In contrast, ERP and MEG directly measure the electrical and magnetic fields generated by neural activity and so have excellent temporal resolution. For the present purposes, the main advantage of these techniques is their ability (in contrast to fMRI) to measure attentional modulation at relatively early stages in categoryspecific visual processing, unconfounded from effects that may occur much later in processing.

Liu and Kanwisher [52] used MEG to ask whether the modulation of face processing by attention described in the previous sections is detectable in the initial stages of face-specific processing revealed by the M170 response (see also [27,28,38]). As described above, they first localized in each subject the face-selective SOIs over occipitotemporal regions in each hemisphere. They then measured the latency and amplitude of the face-selective M170 while subjects carried out the 


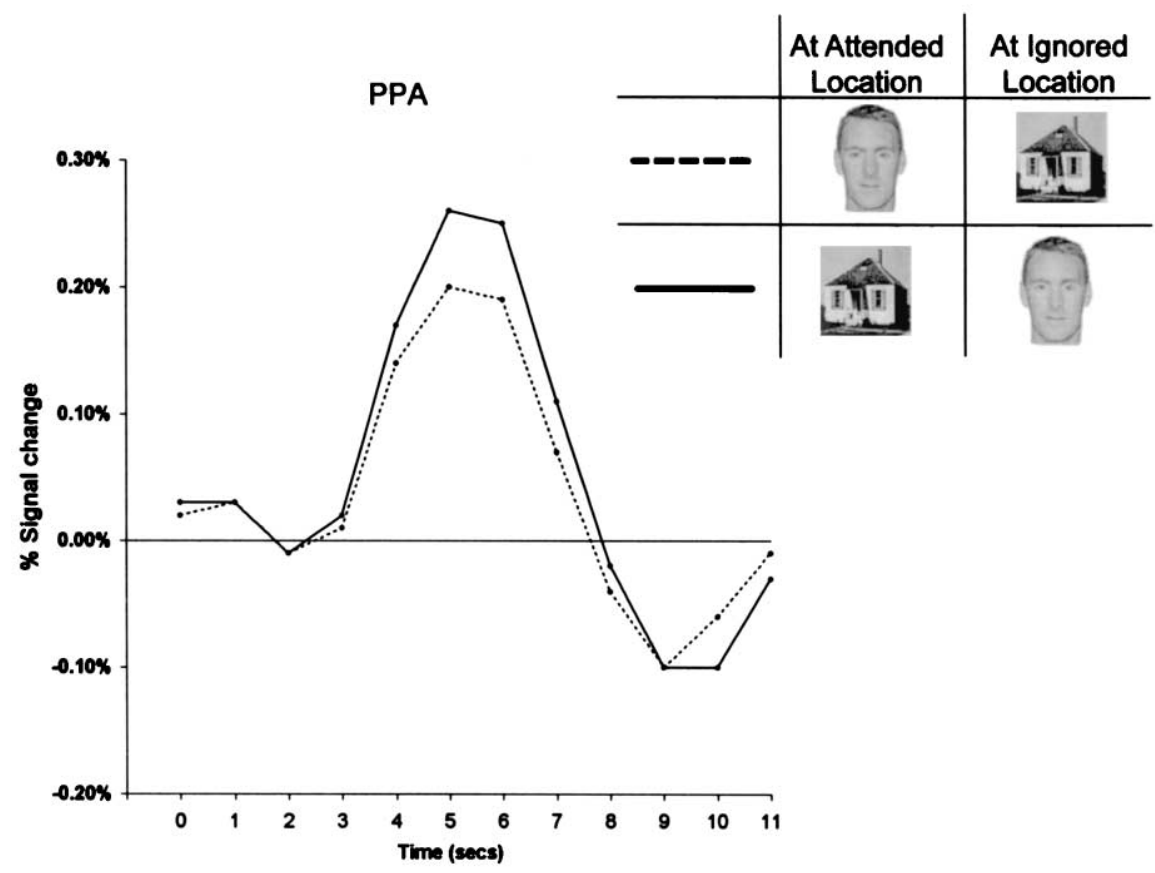

Fig. 7. Results from an fMRI test of location-based attention [22]. The mean event-related evoked response in the PPA was greater when a house occupied the same location as the attended oval, as compared to a face.

following task. On each trial, subjects viewed a single face or a single house, the cue, for $200 \mathrm{~ms}$. After an interval of $800 \mathrm{~ms}$ they were then presented with an overlapping face-house stimulus (see Fig. 8). The subjects' task was to report whether the face or house cue appeared in the compound stimulus. Thus the subjects' attention was directed by the cue to search for either a specific face or a specific house in the compound stimulus.

Fig. 9 shows the grand mean response in each condition, averaged across subjects and hemispheres, and time-locked to the onset of the compound stimulus. No significant effect of attention was found on the latency of the M170 response. Critically, however, the amplitude of the M170 evoked by the overlapping stimuli was significantly larger when subjects were attending to the face than when subjects were attending to the house. These results indicate attentional modulation of the response to faces relatively early in the processing sequence, most likely before feedback from later processes (such as response selection) could take place. Presumably, because the face and house stimuli were superimposed in the same location, this effect does not reflect the early spatial attention mechanisms that have been described extensively in ERP research [36,54].

Might the observed modulation of the M170 have been due to a stimulus-driven aftereffect of the cue, rather than attention per se? We think this unlikely for three reasons. First, the cue and target stimuli were separated far enough in time $(800 \mathrm{~ms})$ that the M170 responses to the two events themselves did not overlap.
Furthermore, repetition of face stimuli has been shown in previous work [40] to reduce the amplitude of the evoked response. Thus in the present paradigm the stimulus effects from the face cues would be expected, if anything, to lower the response to the face in the subsequent target stimulus, in contrast to the enhancement observed. Finally, an informal pilot study in which subjects passively viewed the stimuli from the present experiment revealed no modulation of the response to the M170 as a function of the nominal 'cue' stimulus.

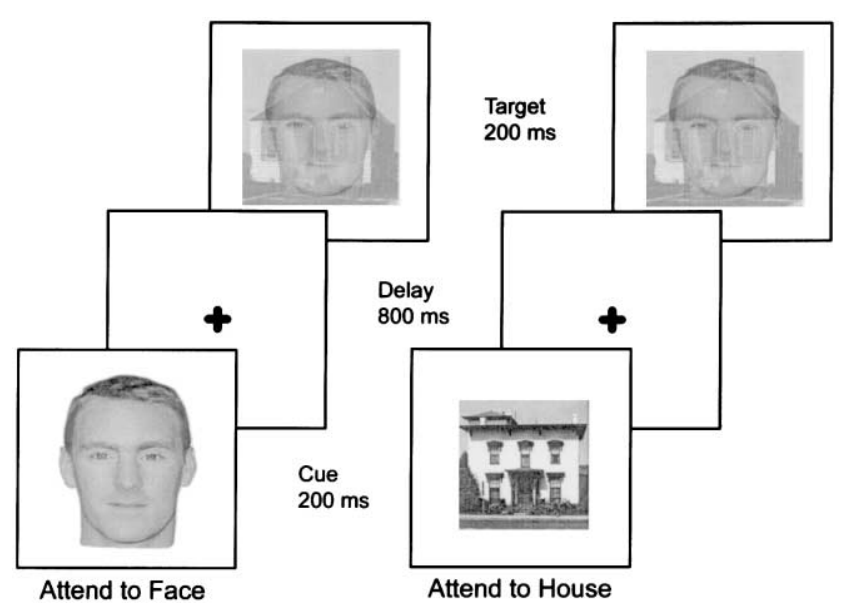

Fig. 8. Illustration of the procedure for an MEG investigation of attentional modulation of face-specific M170 responses [52]. A cue stimulus directed attention either to the face or to the house in the subsequent compound stimulus. Subjects pressed a key whenever the cue stimulus matched the corresponding figure in the compound. 


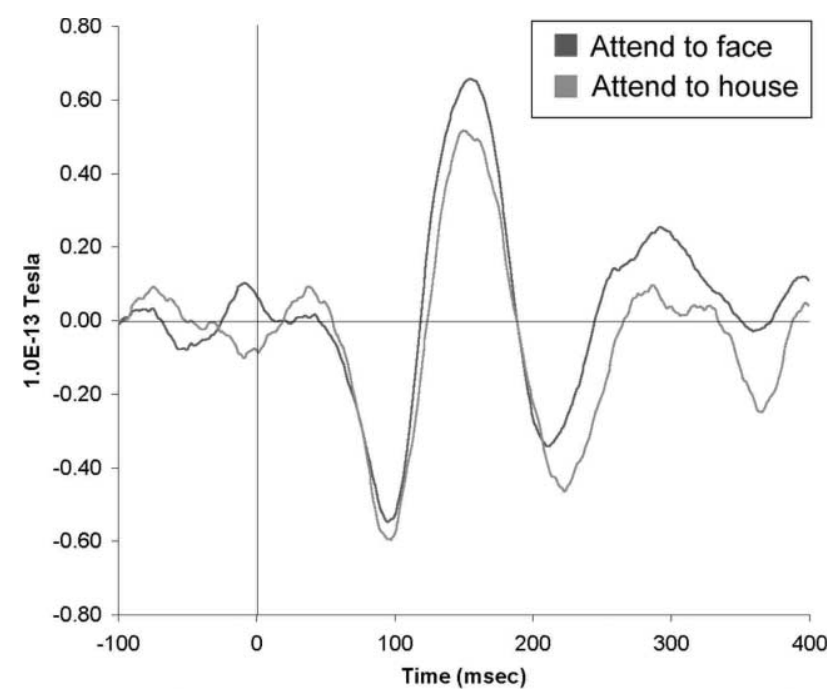

Fig. 9. MEG data showing significant enhancement of the amplitude of the face-specific M170, averaged across the left and right hemisphere, as a result of attention to faces as compared to houses.

Attentional modulation observed at a latency of 170 $\mathrm{ms}$ is unlikely to be a result of decision- or response-related processes occurring after the compound stimulus is initially processed. Thus modulation of face processing, at least in the paradigm tested here, apparently does not depend on later top-down feedback, but can affect a relatively early phase of stimulus-specific processing. Further work using similar techniques will be useful to address whether this modulation is caused by a baseline increase in face-specific cortical areas, initiated in the interval between cue and target, or rather an online modulation of face processing, initiated at the onset of the target stimulus.

\subsection{Control of attention}

The discussion so far has focused on the use of category-specific visual cortical areas (or MEG responses) to measure how stimulus processing is affected by attention. In this final section we review a recent study [88] that asks not about the effects of attention on perceptual processing, but instead about the cortical mechanisms involved in controlling attention (see also $[38,64])$.

The hundreds of published papers on attention make use of a wide variety of tasks designed to tax visual attention, from reporting the identities of letters to the side of fixation, to searching an array of colored shapes for the one green square, to watching a very rapid sequence of words presented at the center of gaze while monitoring for the occurrence of a particular word. The very heterogeneity of tasks used to study attention raises the important question of whether there is anything in common among each of these diverse 'attentional' tasks. Or is attention a fiction, a disjunctive set of unrelated mechanisms that have nothing in common except that they are all called 'attention tasks'? In his book The Psychology of Attention, Pashler [67] argues that "no one knows what attention is, and ...there may not even be an'it' there to be known about".

fMRI provides a way to test whether there is an 'it' of visual attention. Wojciulik and Kanwisher [88] reasoned that if there is a common mechanism that is invoked by a wide variety of attention-requiring tasks, then it should evoke activity in a common brain region. To test this idea, subjects were scanned while carrying out three substantially different attention-requiring tasks. In each, an attentionally-demanding task was compared with a less-demanding version that could be carried out on the same stimuli. All six resulting conditions were tested in each subject in a single scanning session. The key question was whether there were any voxels for which a significant effect of attention would be found for all of the three different attentional tasks. Such an overlapping activation across diverse attentional tasks would imply the existence of a common cortical mechanism engaged by each [see also [71]].

In the first task, subjects monitored a display for changes in the size of a dot. In the attention-demanding version, subjects fixated a central cross-shaped cue. Its orientation indicated which of several peripheral dots, arranged in a circle around fixation, to attend. The cue directed attention to a different dot every $4 \mathrm{~s}$, requiring regular shifts of spatial attention. In the easy version of the same task, which did not require spatial shifts of attention, subjects attended a central dot and monitored it for a size change.

The second task was a modified version of that used in the first study reviewed here ([87]; see Fig. 2). In the difficult version, subjects matched the faces or houses as in the previous study. The easy version of the task required subjects to determine whether the two arms of the central fixation cross were of the same or different color.

Finally, the third task involved monitoring a rapid stream of letters for a target letter defined either by a conjunction of features (the attentionally-demanding version) or a single feature (the easy version; [79]). No spatial shifts of attention were demanded by either task; they differed only in the difficulty of extracting the target items from the stream.

Within each pair of tasks, the stimulus and response conditions for the easy and attentionally-demanding versions were identical. Thus for each task, a comparison of the fMRI data between attentionally-demanding and easy versions could reveal the activations specific to the attention demands of that task. More importantly, any cortical regions showing overlapping activation across all three tasks would constitute a neural signature of a candidate general mechanism for directing attention. 
The results showed significant areas of overlap in most subjects in the region of the IPS (inferior parietal sulcus) (see also [18]). This result is consistent with a cortical area involved in a general fashion in visual selection. However, it is also possible that any difficult task would activate the same region, when compared to a matched easy version of the same task. Wojciulik and Kanwisher [88] tested an additional control condition run in the same session, in which subjects had to report (in the demanding version) whether a visually-presented word was both a noun and a verb (e.g. 'record'). In the easy version of this task, subjects determined for the same stimuli whether the initial and final letters of the word were of the same or different height. In this control task, although large activations were found elsewhere in the brain, no difference in activation was observed in the IPS between the hard and easy versions. This result rules out the possibility that this area is activated generally by any difficult visual task.

The emphasis of this chapter has been on addressing cognitive questions with neuroimaging techniques. How is the Wojciulik and Kanwisher [88] study relevant to this goal? First, these findings suggest that visual attention is not simply a collection of distinct special-purpose mechanisms. Instead, a unitary general-purpose mechanism for directing visual attention may exist. Second, the third task described above (monitoring a stream of letters at fixation) involved little or no spatial component, and no requirement to plan or make eyemovements. Further, the two versions of this task were identical in their requirement to maintain fixation, and in the absence of peripheral stimuli that might encourage saccades. Thus these shared parietal attentional mechanisms need involve neither an eye movement component (generation or suppression), nor a spatial component. In future research it may be possible to capitalize on this apparently process-specific region as a marker for determining the degree to which attention is engaged by various cognitive tasks.

Finally, Wojciulik and Kanwisher's [88] study focused on the contribution of parietal systems to the control of attention. Similar approaches may be useful in characterizing the general involvement of other cortical regions (e.g. frontal cortex) to the control of attention.

\section{General discussion}

\subsection{Methodological issues}

We have made the case that ROI-based approaches provide one powerful way to use the strengths of fMRI and MEG to address psychological hypotheses about attention. This approach obviates many of the concerns that arise in whole-brain subtraction or regression- based analyses. In particular, the need to perform statistical corrections for Type I error is eliminated by focusing analyses on pre-defined voxels. By defining ROIs in each individual, the problems of anatomical variability across individuals, and of variability in the mapping between function and anatomy, are sidestepped. And finally, independent identification of ROIs allows a statistically independent quantification of the magnitude of response in those ROIs, which is essential for testing for the effects of attentional modulation. Although the magnitude of response can be determined in a variety of other ways that do not require ROIs (e.g. regression coefficients), such techniques tend to be biased because the data used in quantifying the response magnitude are not statistically independent from the data used to select the voxels in which this quantification is carried out.

ROI designs are not appropriate for all kinds of neuroimaging study; they are not well-suited, for example, to identifying whole networks of interrelated areas involved in the performance of complex tasks. The rationale for the ROI method presumes a substantial amount of prior evidence for the functional selectivity of a cortical region in processing a particular stimulus type, or in contributing to a particular aspect of task performance. To be tested in individual subjects, which is advantageous for the reasons mentioned above, regions of interest must be sufficiently selective and have sufficient spatial extent to be detected without group averaging. (ROIs can, of course, be defined in a common space over the average of a group of subjects. This will tend to be accompanied, however, in reduced selectivity of the voxels identified). Finally, ROI methods do not preclude the use of additional, exploratory analyses on the same data set; there is no reason not to see how the rest of the brain regions scanned responded in the same contrasts.

\subsection{Conclusions}

We have used fMRI and MEG markers of face processing, and fMRI markers of place processing, as online measures of the effects of selective attention on visual information processing. These studies revealed converging evidence for attentional modulation of face processing, and showed that this modulation, at least in some circumstances, can occur relatively early in the processing sequence. Further experiments isolated specific location-based and object-based mechanisms of attentional selection. Finally, we illustrated how the approach developed here can go beyond simply showing the effects of attention on stimulus representation, to reveal the common mechanisms involved in a wide variety of attentionally-demanding tasks.

We believe the approach we have outlined here could be generalized in many ways to fruitfully study a wide 
range of questions about attention [45]. ROIs of course need not be restricted to high-level category-specific regions. For example, several recent studies have used fMRI to demonstrate the powerful effects of attention in the precise region of primary visual cortex where the critical stimulus is processed $[33,75,77]$. Further, imaging studies have revealed the fMRI signatures of nonvisual sensory processing. For example, recent evidence suggesting the existence of human-voice specific regions of auditory cortex [6] could serve as the analogue of the FFA and PPA in studies of auditory attention. In principle, any cortical area that produces a reliable stimulus-specific response could be used to measure the effects of varying attentional manipulations on processing of that stimulus. There are many similarities between the methods developed here and single-unit neurophysiology, with the region of interest (and its preferred and non-preferred stimuli) playing the role of the single neuron.

Studies on the control of attention $[15,16,38,62,70,88]$ are only just beginning to parse the functional components of the attentional control system. Neuroimaging may enable us to determine whether common or distinct mechanisms are involved in overt and covert attention [17,63], endogenous and exogenous attention, and to distinguish between more fine-grained components of the attentional system.

Substantial challenges remain. Can neuroimaging studies provide converging evidence on some of the key theoretical debates in the field? For example, feature integration theory $[79,80]$ and the biased-competition model [21] represent alternative frameworks that interpret the same data (e.g. search rates in visual search tasks) in terms of substantially different mechanisms. Perhaps neuroimaging will some day be able to distinguish between such theories, an achievement that would represent a major contribution. Likewise, the promise of cognitive neuroscience is that findings from different methodologies will cross-fertilize. Neuroimaging techniques will further prove their usefulness to the extent that they suggest crucial new behavioral or neuropsychological investigations. Finally, one of the greatest limitations in current neuroimaging research concerns spatial and temporal resolution: while we can now obtain good resolution in either space or time, we do not have methods for achieving both at once with noninvasive techniques. However, ongoing research [19] is attempting to combine fMRI and MEG data to provide the ultimate data for human cognitive neuroscience: the precise, high-resolution time course of activity in each voxel in a normal human brain [19]. If successful, these techniques could provide the most powerful tool yet for investigating the mechanisms underlying visual attention.

\section{Acknowledgements}

Portions of this research were supported by a Human Frontiers grant to NK. We thank Ravi Menon, Joe Gati, Ewa Wojciulik, and Jody Culham for assistance with scanning at UWO.

\section{References}

[1] Aguirre GK, Zarahn E, D'Esposito M. An area within human ventral cortex sensitive to 'building' stimuli: evidence and implications. Neuron 1998;21:373-83.

[2] Allison T, Ginter H, McCarthy G, Nobre AC, Puce A, Luby M, Spencer DD. Face recognition in human extrastriate cortex. Journal of Neurophysiology 1994;71:821-5.

[3] Allison T, Puce A, Spencer DD, McCarthy G. Electrophysiological studies of human face perception I: potentials generated in occipitotemporal cortex by face and non-face stimuli. Cerebral Cortex 1999;9:415-30.

[4] Baylis GC, Driver J. Visual parsing and response competition: the effect of grouping factors. Perception and Psychophysics 1992;51:145-62.

[5] Beauchamp MS, Cox RW, DeYoe EA. Graded effects of spatial and featural attention on human area MT and associated motion processing areas. Journal of Neurophysiology 1997;78:516-20.

[6] Belin P, Zatorre RJ, Philippe L, Ahad P, Pike B. Voice-selective areas in human auditory cortex. Nature 2000;403:309-12.

[7] Bentin S, Allison T, Puce A, Perez E, McCarthy G. Electrophysiological studies of face perception in humans. Journal of Cognitive Neuroscience 1996;8:551-65.

[8] Blaser E, Pylyshyn Z, Holcombe A. Tracking an object through feature-space. Nature 2000;408:196-9.

[9] Braun J. Intimate attention. Nature 2000;408:154-5.

[10] Brefczynski JA, DeYoe EA. A physiological correlate of the 'spotlight' of visual attention. Nature Neuroscience 1999;4:3704.

[11] Broadbent D. Perception and Communication. London: Pergamon Press, 1958.

[12] Connor CE, Gallant JL, Preddie DC, Van Essen DC. Responses in area V4 depend on the spatial relationship between stimulus and attention. Journal of Neurophysiology 1996;75:1306-8.

[13] Connor CE, Preddie DC, Gallant JL, Van Essen DC. Spatial attention effects in macaque area V4. Journal of Neuroscience 1997;17:3201-14.

[14] Corbetta M, Miezin FM, Dobmeyer S, Shulman GL, et al. Attentional modulation of neural processing of shape, color, and velocity in humans. Science 1990;248:1556-9.

[15] Corbetta M, Miezin FM, Shulman GL, Petersen SE. A PET study of visuospatial attention. Journal of Neuroscience 1993; 13:1202-26.

[16] Corbetta M, Shulman GL, Miezin FM, Petersen SE. Superior parietal cortex activation during spatial attention shifts and visual feature conjunction. Science 1995;270:802-5.

[17] Corbetta M, Akbudak E, Conturo TE, Snyder AZ, Ollinger JM, Drury HA, Linenweber MR, Petersen SE, Raichle ME, Van Essen DC, Shulman GL. A common network of functional areas for attention and eye movements. Neuron 1998;21:761-73.

[18] Coull JT, Frith CD. Differential activation of right superior parietal cortex and intraparietal sulcus by spatial and nonspatial attention. Neuroimage 1998;8:176-87.

[19] Dale AM, Liu AK, Fischl BR, Buckner RL, Belliveau JW, Lewine JD, Halgren E. Dynamic statistical parametric mapping: combining fMRI and MEG for high-resolution imaging of cortical activity. Neuron 2000;26:55-67. 
[20] Davis G, Driver J, Pavani F, Shepard A. Reappraising the apparent costs of attending to two separate visual objects. Vision Research 2000;40:1323-32.

[21] Desimone R, Duncan J. Neural mechanisms of selective visual attention. Annual Review of Neuroscience 1995;18:193-222.

[22] Downing P, Kanwisher N. fMRI evidence for location-based attentional selection. Talk presented at the Annual Meeting of the Society for Neuroscience. New Orleans, LA, 2000.

[23] Driver J, Baylis GC. Movement and visual attention: the spotlight metaphor breaks down. Journal of Experimental Psychology: Human Perception and Performance 1989;15:448-56.

[24] Duncan J. Selective attention and the organization of visual information. Journal of Experiment Psychology: General 1984;113:501-17.

[25] Egly R, Driver J, Rafal RD. Shifting visual attention between objects and locations: evidence from normal and parietal lesion subjects. Journal of Experimental Psychology: General 1994;123:161-77.

[26] Eimer M. 'Sensory gating' as a mechanism for visuospatial orienting: electrophysiological evidence from trial-by-trial cuing experiments. Perception and Psychophysics 1994;55:667-75.

[27] Eimer M. Attentional modulations of event-related brain potentials sensitive to faces. Cognitive Neuropsychology 2000;17:10316.

[28] Eimer M. Crossmodal links in spatial attention between vision, audition, and touch: evidence from event-related brain potentials. Neuropsychologia 2001;39:1292-1303.

[29] Epstein R, Kanwisher N. A cortical representation of the local visual environment. Nature 1998;392:598-601.

[30] Epstein R, Stanley D, Harris A, Kanwisher N. The parahippocampal place area: perception, encoding, or memory retrieval? Neuron 1999;23:115-25.

[31] Eriksen CW, St. James JD. Visual attention within and around the field of focal attention: a zoom lens model. Perception and Psychophysics 1986;40:225-40.

[32] Farah MJ, Wilson KD, Drain HM, Tanaka JR. The inverted face inversion effect in prosopagnosia: evidence for mandatory, face-specific perceptual mechanisms. Vision Research 1995;35:2089-93.

[33] Gandhi SP, Heeger DJ, Boynton GM. Spatial attention affects brain activity in human primary visual cortex. Proceedings of the National Academy of Sciences USA 1999;96:3314-9.

[34] Halgren E, Raij T, Marinkovic K, Jousmaki V, Hari R. Cognitive response profile of the human fusiform face area as determined by MEG. Cerebral Cortex 2000;10:69-81.

[35] Hillyard SA, Hink RF, Schwent VL, Picton TW. Electrical signs of selective attention in the human brain. Science 1973;182:17780.

[36] Hillyard SA, Mangun GR, Woldorff MG, Luck SJ. Neural systems mediating selective attention. In: Gazzaniga MS, editor. The Cognitive Neurosciences. Cambridge, MA: MIT Press, 1995:665-82.

[37] Hoffman JE, Nelson B. Spatial selectivity in visual search. Perception and Psychophysics 1981;30:283-90.

[38] Hopfinger B, Woldorff MG, Fletcher EM, Mangun GR. Dissociating top-down attentional control from selective perception and action. Neuropsychologica 2001;39:1277-1291.

[39] Ishai A, Ungerleider LG, Martin A, Schouten JL, Haxby JV. Distributed representation of objects in the human ventral visual pathway. Proceedings of the National Academy of Sciences USA 1999;96:9379-84.

[40] Jeffreys DA. Evoked potential studies of face and object processing. Visual Cognition 1996;3:1-38.

[41] Kahneman D, Treisman A, Gibbs BJ. The reviewing of object files: object-specific integration of information. Cognitive Psychology 1992;24:175-219.
[42] Kanwisher N, Driver J. Objects, attributes, and visual attention. Current Directions in Psychological Science 1992;1:26-31.

[43] Kanwisher N, McDermott J, Chun M. The fusiform face area: a module in human extrastriate cortex specialized for perception of faces. Journal of Neuroscience 1997;17:4302-11.

[44] Kanwisher N. Domain specificity in face perception. Nature Neuroscience 2000;3:759-63.

[45] Kanwisher N, Wojciulik E. Visual attention: insights from brain imaging. Nature Reviews: Neuroscience 2000;1:91-100.

[46] Kanwisher N, Downing P, Epstein R, Kourtzi Z. Functional neuroimaging of human visual recognition. In: Cabeza R, Kingstone A, editors. Handbook of Functional Neuroimaging of Cognition. Cambridge, MA: MIT Press, 2001.

[47] Kastner S, Pinsk MA, DeWeerd P, Desimone R, Ungerleider LG. Increased activity in human visual cortex during directed attention in the absence of visual stimulation. Neuron 1999;22:751-61.

[48] Kastner S, Ungerleider LG. The neural basis of biased competition in human visual cortex. Neuropsychologia 2001;39:12631276.

[49] LaBerge D, Brown V. Variations in size of the visual field in which targets are presented: an attentional range effect. Perception and Psychophysics 1986;40:188-200.

[50] Lamme VF, Roelfsma PR. The distinct modes of vision offered by feedforward and recurrent processing. Trends in Neuroscience 2000;23:571-9.

[51] Liu J, Higuchi M, Marantz A, Kanwisher N. Magnetophysiological studies of face and non-face processing. Neuroreport 2000;11:337-41.

[52] Liu J, Kanwisher N. Covert visual attention modulates face-specific activity at $160 \mathrm{msec}$ : An MEG study. Poster presented at the annual meeting of the Society for Cognitive Neuroscience. San Francisco, 2000.

[53] Luck SJ, Chelazzi L, Hillyard SA, Desimone R. Neural mechanisms of spatial selective attention in areas V1, V2, and V4 of macaque visual cortex. Journal of Neurophysiology 1997;77:2442.

[54] Luck SJ, Woodman GF, Vogel EK. Event-related potential studies of attention. Trends in Cognitive Science 2000;4:432-40.

[55] Mack A, Rock I. Inattentional Blindness. Cambridge, MA: MIT Press, 1998.

[56] Marois R, Leung HC, Gore JC. A stimulus-driven approach to object identity and location processing in the human brain. Neuron 2000;25:717-28.

[57] Martinez A, Anllo-Vento L, Sereno MI, Frank LR, Buxton RB, Dubowitz DJ, Wong EC, Hinrichs H, Heinze HJ, Hillyard SA. Involvement of striate and extrastriate visual cortical areas in spatial attention. Nature Neuroscience 1999;2:364-9.

[58] McCarthy G, Puce A, Gore JC, Allison T. Face-specific processing in the human fusiform gyrus. Journal of Cognitive Neuroscience 1997;9:604-9.

[59] McCarthy G, Puce A, Belger A, Allison T. Electrophysiologcial studies of human face perception II: Response properties of face-specific potentials generated in occipitotemporal cortex. Cerebral Cortex 1999;9:431-44.

[60] Moran J, Desimone R. Selective attention gates visual processing in the extra-striate cortex. Science 1985;229:782-4.

[61] Motter BC. Focal attention produces spatially selective processing in visual cortical areas V1, V2, and V4 in the presence of competing stimuli. Journal of Neurophysiology 1993;70:909-19.

[62] Nobre AC, Sebestyn GN, Gitelman DR, Mesulam M-M, Frackowiak RS, Frith CD. Functional localization of the system for visuospatial attention using positron emission tomography. Brain 1997;120:515-33.

[63] Nobre AC, Gitelman DR, Dias EC, Mesulam MM. Covert visual spatial orienting and saccades: overlapping neural systems. Neuroimage 2000;11:210-6. 
[64] Nobre AC. Orienting attention to instants in time. Neuropsychologia 2001;39:1317-1328.

[65] O'Craven KM, Rosen BR, Kwong KK, Treisman A, Savoy RL. Voluntary attention modulates fMRI activity in human MTMST. Neuron 1997;18:591-8.

[66] O'Craven K, Downing P, Kanwisher N. fMRI evidence for objects as the units of attentional selection. Nature 1999;401:584-7.

[67] Pashler HE. The Psychology of Attention. Cambridge, MA: MIT Press, 1998.

[68] Posner MI. Orienting of attention. Quarterly Journal of Experimental Psychology 1980;32:3-25.

[69] Posner MI, Snyder CRR, Davidson BJ. Attention and the detection of signals. Journal of Experimental Psychology: General 1980;109:160-74.

[70] Posner MI, Dehaene S. Attentional networks. Trends in Neuroscience 1994;17:75-9.

[71] Price C, Friston J. Cognitive conjunction: a new approach to brain activation experiments. NeuroImage 1997;5:261-70.

[72] Puce A, Allison T, McCarthy G. Electrophysiological studies of human face perception. III: Effects of top-down processing on face-specific potentials. Cerebral Cortex 1999;9:445-58.

[73] Rees G, Russell C, Frith CD, Driver J. Inattentional blindness versus inattentional amnesia for fixated but ignored words. Science 1999;286:2504-7.

[74] Rees G, Lavie N. What can functional imaging reveal about the role of attention in visual awareness? Neuropsychologia 2001;39:1343-1353.

[75] Ress D, Backus B, Heeger D. Activity in primary visual cortex predicts performance in a visual detection task. Nature Neuroscience 2000;3:940-5.

[76] Shapiro KL, Arnell KM, Raymond JE. The attentional blink. Trends in Cognitive Sciences 1997;1:291-6.
[77] Somers DC, Dale AM, Seiffert AE, Tootell RB. Functional MRI reveals spatially specific attentional modulation in human primary visual cortex. Proceedings of the National Academy of Sciences USA 1999;96:1663-8.

[78] Tarr MJ, Gauthier I. FFA: A flexible fusiform area for subordinate-level visual processing automatized by expertise. Nature Neuroscience 2000;3:764-9.

[79] Treisman A, Gelade G. A feature-integration theory of attention. Cognitive Psychology 1980;12:97-136.

[80] Treisman A. The perception of features and objects. In: Baddeley A, Weiskrantz L, editors. Attention: Selection, Awareness, and Control. Oxford: Clarendon Press, 1993.

[81] Treisman A. The binding problem. Current Opinion in Neurobiology 1996;6:171-8.

[82] Treue S, Martinez Trujillo JC. Feature-based attention influences motion procesing gain in macaque visual cortex. Nature 1999;399:575-9.

[83] Tsal Y, Lamy D. Attending to an object's color entails attending to its location: support for location-special views of visual attention. Perception and Psychophysics 2000;62:960-8.

[84] Vuilleumier P. Faces call for attention: evidence from patients with visual extinction. Neuropsychologia 2000;38:693-700.

[85] Vuilleumier P, Schwartz S. Emotional facial expressions capture attention. Neurology 2001;56:153-8.

[86] Watson SE, Kramer AF. Object-based visual selective attention and perceptual organization. Perception and Psychophysics 1999;61:31-49.

[87] Wojciulik E, Kanwisher N, Driver J. Covert visual attention modulates face-specific activity in the human fusiform gyrus: fMRI study. Journal of Neurophysiology 1998;79:1574-8.

[88] Wojciulik E, Kanwisher N. The generality of parietal involvement in visual attention. Neuron 1999;23:747-64. 